\title{
Adipositas in den USA: Schuld sind die vielen Snacks
}

\begin{abstract}
Die USA sind die Nation der Dicken. Nach Schätzungen werden 2015 drei von vier US-Amerikanern übergewichtig sein. Einer aktuellen Studie zufolge sind die Hauptursache nicht mehr die typischen amerikanischen Riesenportionen (supersize). Vielmehr soll sich jetzt der Trend zu häufigeren Zwischenmahlzeiten (Snacks) ungünstig auf die schlanke Linie auswirken.
\end{abstract}

- Wissenschaftler der University of North Carolina in Chapel Hill verglichen die Menge und die Hauptquelle der täglich konsumierten Kalorien in den vergangenen 30 Jahren. Demnach verzehrte ein US-Amerikaner von 2003 bis 2006 täglich $2374 \mathrm{Kcal}$, das waren 570 mehr als noch zwischen 1977 und 1978. 1977/1978 und 1989-1991 waren die wachsenden Portionen noch der entscheidende Faktor für die zunehmenden Gewichtsprobleme der Amerikaner. Die tägliche Durchschnittszufuhr nahm jährlich um $15 \mathrm{Kcal}$ zu. Die Zahl der Mahlzeiten machte sich mit einem jährlichen Plus von 4 Kcal pro Tag damals noch vergleichsweise wenig bemerkbar.

Später wendete sich das Blatt, die vielen Snacks ließen die Amerikaner runder und runder werden: In der letzten Phase der Untersuchung, zwischen 1994 und 1998 beziehungsweise 2003 und 2006, erhöhte sich die tägliche Kalorienzufuhr pro Jahr um $39 \mathrm{kcal}$. In der gleichen Zeit nahm die Portionsgröße sogar leicht ab, rechnerisch um jährlich eine Kilokalo- rie. Das zeigt, dass die Gewichtszunahme maßgeblich vom Snacken herrührt, nicht mehr von Portionen im XXL-Format.

\section{Kommentar}

Die Resultate suggerieren einen neuen Fokus, auf den die Bemühungen zu konzentrieren sind, um in den USA (und sicher nicht nur dort) die Energiebilanz zu normalisieren.

K. MALBERG =

- K. J. Duffey et al.

(Korrespondenz: BM Popkin, Carolina Population Center, University of North Carolina at Chapel Hill, Chapel Hill, North Carolina, e-mail: popkin@unc.edu) Energy density, portion size, and eating occasions:Contributions to increased energy intake in the United States, 1977-2006. Published online June 28, 2011 in PLoS Med 8(6): e1001050. doi:10.1371/journal. pmed. 1001050

\section{Achtung:}

\section{Hier muss der \\ Dummy durch eine Anzeige ersetzt werden !!}

